\title{
Uma Análise Regional sobre o Perfil de Estudantes Concluintes em cursos STEAM através do ENADE
}

\author{
Jeovana Leão, Adrya Araújo, Bruna Mariana F. de Souza, Julis Figueira de \\ Araújo, Kalil Ferreira, Mariana Barros, Tanara Lauschner,Fabíola Guerra \\ Nakamura
}

\author{
Universidade Federal do Amazonas (UFAM) \\ 69077-000 - Manaus-AM, Brasil \\ \{aadoa bruna.mariana, keff tanara, fabiola\}@icomp.ufam.edu.br \\ \{jeovanaleao, araujojules 7, marianafranco\} @ super.ufam.edu.br
}

\begin{abstract}
In order to investigate the profile of students graduating from courses in the STEAM areas in the state of Amazonas, this article analyzes socioeconomic data of participants in the ENADE (National Student Performance Exam) in the years 2017 to 2019. The results showed that the majority of students still it is male, that most women had characteristics of economic vulnerability and lack of representativeness when gender was associated with the indigenous race. In addition to the permanence of gender stigma associated with motivation to choose a course when compared between men and women in the sample. It is concluded that there is a need to carry out more in-depth analyzes in certain aspects of the research, such as the interconnection of basic education data, however the analysis made in this article verified the indispensability of actions within higher education institutions that contribute to the permanence of students.
\end{abstract}

Resumo. Visando investigar o perfil de estudantes concluintes de cursos nas áreas STEAM no estado do Amazonas, este artigo analisa dados socioeconômicos dos participantes do ENADE (Exame Nacional de Desempenho dos Estudantes) nos anos de 2017 a 2019. Os resultados mostraram que a maioria dos estudantes ainda é masculina, que mulheres em sua maioria apresentavam características de vulnerabilidade econômica e a falta de representatividade quando o gênero foi associado à raça indígena. Além da permanência do estigma de gênero associado a motivação para escolha de curso quando comparada entre os homens e mulheres da amostra. Conclui-se que há a necessidade de realização de análises mais aprofundadas em determinados aspectos da pesquisa, como a interligação de dados do ensino básico, porém a análise feita neste artigo verificou a indispensabilidade de ações dentro de instituições de ensino superior que contribuam para a permanência de estudantes.

\section{Introdução}

O ENADE, Exame Nacional de Desempenho dos Estudantes, é aplicado pelo Inep (Instituto Nacional de Estudos e Pesquisas Educacionais Anísio Teixeira) desde 2004 e avalia o rendimento dos concluintes dos cursos de graduação em relação aos conteúdos programáticos previstos nas diretrizes dos cursos, o desenvolvimento de competências e habilidades necessárias ao aprofundamento da formação geral e profissional, e o nível de atualização dos estudantes com relação à realidade brasileira e mundial ${ }^{1}$. Tendo como candidatos estudantes concluintes de cursos em instituições de ensino superior, os resultados da avaliação de cursos de graduação, avaliação institucional em conjunto

$\overline{1}$ https://www.gov.br/inep/pt-br/areas-de-atuacao/avaliacao-e-exames-educacionais/enade 
com as respostas do Questionário do Estudante, trazem fortes dados indicadores de qualidade da educação superior [Zucatelli, 2019].

Com o objetivo de traçar o perfil de estudantes concluintes dos cursos STEAM (Science, Technology, Engineering, Arts and Mathematics), a análise da base de dados do ENADE mostrou-se uma fonte importante de informações para a realização de um estudo de gênero e identificação de possíveis fatores que dificultam a permanência de estudantes na graduação, questão discutida em outras literaturas. Mas especificamente avaliados os dados dos cursos de Engenharia Elétrica e suas ênfases, Engenharia da Computação, Engenharia de Produção, Design, Ciência da Computação, Engenharia de Software e Sistemas de Informação. No entanto, devido ao ciclo trienal do ENADE - a cada ano um grupo de áreas do conhecimento é avaliado - foram utilizados microdados dos anos 2017-2019 para que todos os cursos STEAM em questão na pesquisa deste artigo fossem abrangidos.

O artigo está organizado da seguinte forma. Na Seção 2, são mostrados os trabalhos pesquisados que possuem relação com ENADE. Na Seção 3, consta a metodologia utilizada na pesquisa e as variáveis consideradas. Na Seção 4, são apresentadas as análises e resultados da pesquisa feita através dos microdados disponibilizados pelo ENADE. Na Seção 5, temos considerações para futuras pesquisas, concluindo na Seção 6.

\section{Trabalhos Relacionados}

Existem trabalhos que trazem a abordagem de análise dos microdados do Inep, referentes ao ENADE, para estudo de perfil socioeconômico de estudantes de graduação e pós-graduação. Dentre alguns desses trabalhos o fator gênero/sexo é ressaltado, ponto que foi considerado na pesquisa de bibliografias relacionadas neste artigo.

Freitas et al (2019) destacam que dentre as pessoas utilizadas na amostra de sua pesquisa nos cursos da área de computação, a maioria das mulheres afirmou que o principal motivo para escolher o curso foi a inserção no mercado de trabalho, diferindo da afirmação da maioria dos homens que apontaram como motivo de escolha de curso a vocação, fator que reforça a existência do estigma de que mulheres não possuem vocação para a área de exatas. Carvalhaes et al (2019) apontam esse estigma como uma forte estratificação por gênero e que diferenças nas escolhas de homens e mulheres dependem de diferenças culturais e sociais que pessoas de sexos diferentes encontram ao longo da vida.

Nasu (2020), em seu estudo relacionando sexo e desempenho acadêmico na educação contábil, sustenta que os fatores socioeconômicos e acadêmicos precisam ser oportunizados aos discentes homens e mulheres de forma equivalente com o objetivo de possibilitar chances mais igualitárias de performance. Observou-se que as alunas estavam mais associadas a faixas de renda familiar e de horas semanais de estudo reduzidas, além do estado civil de casada.

Colombo (2018), em seu estudo sobre a inserção de mulheres nos cursos de pósgraduação no Brasil, verificou que embora as mulheres sejam mais da metade dos ingressantes dos cursos de graduação, a participação feminina nos cursos de pósgraduação é inferior àquela verificada entre o grupo de concluintes dos cursos, principalmente nas grandes áreas de engenharias, ciências exatas e da terra. Zuccarelli (2019), por outro lado mostra que há forte representação feminina entre os concluintes das licenciaturas, assim como nas áreas associadas à Saúde e Bem-Estar.

Tomás (2019) identificou que as estratificações horizontais são cruciais para se compreender o rumo das desigualdades persistentes que surgem e se reproduzem na educação e têm consequências no mercado de trabalho e em outros aspectos no ciclo de vida. 
Um diferencial do presente artigo se dá na não correlação dos fatores socioeconômicos dos candidatos ao seu desempenho. Nesse contexto, o objetivo é aprofundar a pesquisa voltada para concluientes nas áreas STEAM no estado do Amazonas, analisando fatores socioeconômicos, relacioná-los ao sexo e assim verificar possíveis correlações apresentadas em outras literaturas.

\section{Metodologia}

Os microdados do ENADE são disponibilizados pelo Inep. Esses dados podem ser obtidos via download, em formato ASCII, e contêm inputs para leitura por meio dos softwares SAS e SPSS ${ }^{2}$. Os arquivos são organizados por ano e cada um contém microdados referentes a 137 variáveis identificadas pelos dicionários de variáveis de cada ano. Essas variáveis trazem informações relativas à instituição de ensino superior, ao curso, ao estudante e à prova. Fizemos uma seleção destas variáveis baseada nos objetivos do trabalho. Elas estão listadas na Tabela 1.

\section{Tabela 1. Agentes de análise utilizados na pesquisa}

\begin{tabular}{|l|}
\hline \multicolumn{1}{|c|}{ Agentes de análise correspondentes utilizados } \\
\hline Tipo de instituição; \\
\hline Curso; \\
\hline Município e estado; \\
\hline Sexo; \\
\hline Cor/raça; \\
\hline Renda mensal; \\
\hline Tipo de escola de formação no ensino médio; \\
\hline Motivação para cursar e permanecer na graduação; \\
\hline Tipos de bolsa de estudo ou financiamento recebido. \\
\hline
\end{tabular}

Os recortes analíticos dos arquivos foram feitos utilizando a linguagem de programação Python3 na plataforma de kernels, Jupyter Notebook.

A amostra de pesquisa foi formada por 1619 estudantes, todos do estado do Amazonas, sendo $415(25,6 \%)$ do sexo feminino e $1204(74,4 \%)$ do sexo masculino. O percentual por curso é mostrado na Tabela 2.

Para avaliação dos estudantes dos cursos de Engenharia Elétrica e suas ênfases, Engenharia da Computação e Engenharia de Produção foram analisados os microdados do ano de 2019, para avaliação do curso de Design foram analisados os microdados do ano de 2018 e para avaliação dos cursos de Ciência da Computação, Engenharia de Software e Sistemas de Informação foram analisados os microdados do ano de 2017. Também foram considerados dados do IBGE (Instituto Brasileiro de Geografia e Estatistica) analisados a partir do Sistema IBGE de Recuperação Automática (SIDRA) para obter informações quanto ao perfil socioeconômico da população do estado em questão. Todos os microdados estudados correspondem aos dados mais recentes referentes aos cursos considerados na pesquisa deste artigo.

2 https://www.gov.br/inep/pt-br/acesso-a-informacao/dados-abertos/microdados 
Tabela 2. Percentuais de participantes do ENADE considerados nas análises

\begin{tabular}{|c|c|c|}
\hline Curso & $\begin{array}{c}\text { Percentual de estudantes do } \\
\text { sexo feminino (\%) }\end{array}$ & $\begin{array}{c}\text { Percentual de estudantes } \\
\text { do sexo masculino (\%) }\end{array}$ \\
\hline Engenharia da Computação & 15,10 & 84,90 \\
\hline Engenharia Elétrica & 21,19 & 78,81 \\
\hline Engenharia Elétrica - Eletrotécnica & 36,70 & 63,30 \\
\hline Engenharia Elétrica - Eletrônica & 19,00 & 81,00 \\
\hline Engenharia Elétrica - Telecomunicações & 29,40 & 70,00 \\
\hline Engenharia de Produção & 38,50 & 61,50 \\
\hline Engenharia de Software & 15,00 & 85,00 \\
\hline Ciência da Computação & 15,10 & 84,90 \\
\hline Design & 48,00 & 52,00 \\
\hline Sistemas de Informação & 17,30 & 82,70 \\
\hline
\end{tabular}

\section{Resultados}

A pesquisa presente neste artigo considerou o sexo e o tipo de instituição dos participantes correlacionados a cada um dos seguintes agentes de análise: curso, cor/raça, renda mensal e tipo de escola de formação do ensino médio. Do total de 1619 estudantes, 157 do sexo feminino e 301 do sexo masculino graduam em instituições públicas bem como 258 do sexo feminino e 903 do sexo masculino graduam em instituições privadas. Pode-se perceber que os resultados da análise mostraram que a maioria dos estudantes da amostra graduam em instituição privada $(71,7 \%)$ e são do sexo masculino $(74,4 \%)$.

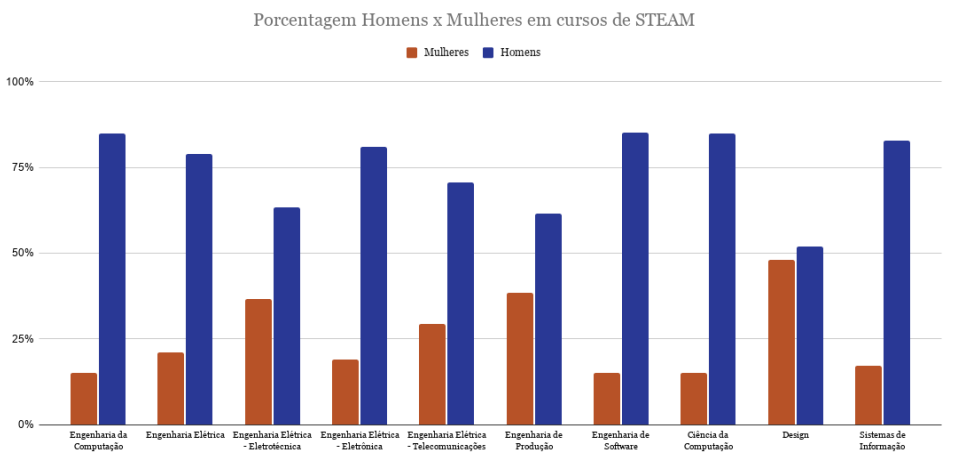

Figura 1. Percentual de mulheres e homens em cursos STEAM

As Figuras 2 e 3 mostram o percentual de pessoas do sexo feminino e masculino nos cursos STEAM, analisados nessa pesquisa, em instituições públicas e privadas respectivamente. Observa-se que independentemente do tipo de instituição o percentual de pessoas do sexo masculino sempre é maior, com exceção do curso de Design em instituição pública que apresenta $66,1 \%$ de pessoas do sexo feminino. Em instituição pública destaca-se o percentual masculino no curso de Engenharia de Software (85\%) e em instituição privada no curso de Engenharia da Computação (87,5\%), além de Design em instituição privada que apresenta maior percentual feminino dentre os cursos $(37,2 \%)$. 


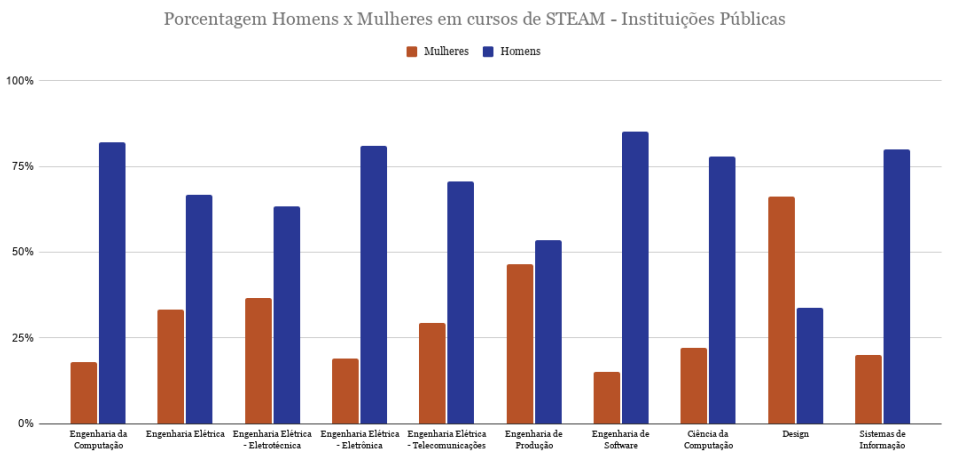

Figura 2. Percentual de mulheres e homens por curso em instituições públicas

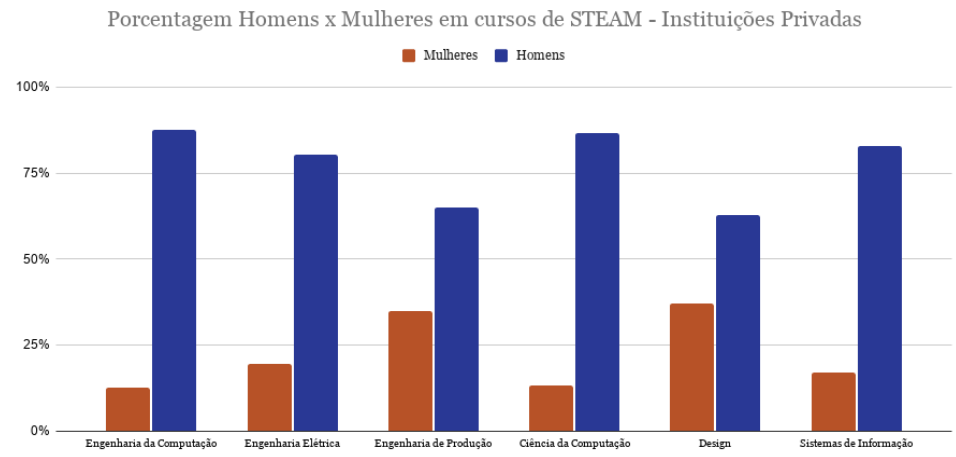

Figura 3. Percentual de mulheres e homens por curso em instituições privadas

Nas Figuras 4 e 5 são apresentados os percentuais de pessoas do sexo feminino e masculino classificadas por cor/raça em instituições públicas e privadas respectivamente. É visto que a maioria dos estudantes, independente de instituição e sexo se declara parda, além disso, observa-se que o percentual de pessoas que se autodeclaram brancos é maior em instituições públicas $(25,5 \%$ do sexo feminino e $22,3 \%$ do sexo masculino) e o percentual de pessoas que se autodeclaram pretos é maior em instituições privadas (4,7\% do sexo feminino e 6,6\% do sexo masculino).

Segundo dados do último censo demográfico (2010) a população residente na unidade de federação Amazonas classificada por cor/raça compõe em maioria pardos(as) $(68,88 \%)^{3}$, proporção que se equipara a da amostra utilizada na pesquisa. Em contrapartida, o percentual de pessoas indígenas em instituições públicas $(0 \%$ do sexo feminino e $0,3 \%$ do sexo masculino) se mostrou muito baixo se comparado a população indígena no estado do Amazonas que chega a 4,84\% ${ }^{3}$. 


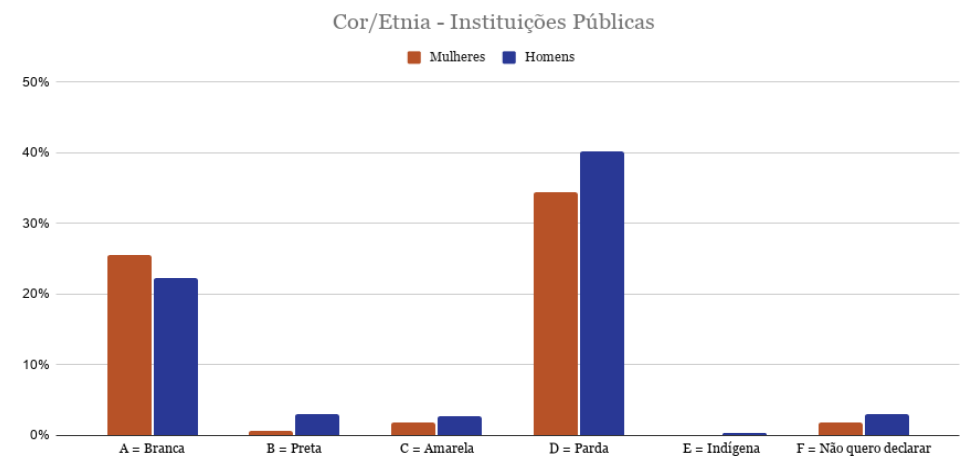

Figura 4. Percentual de mulheres e homens por cor/etnia em instituições públicas



Figura 5. Percentual de mulheres e homens por cor/etnia em instituições privadas

O percentual de pessoas do sexo feminino e masculino categorizadas por renda mensal é ilustrado nas Figura 6 e 7 em instituições públicas e privadas respectivamente. Independentemente do tipo de instituição, é perceptível que o maior percentual de estudantes soma renda familiar e individual mensal de até 1,5 salário mínimo, porém, se comparado o percentual entre os sexos analisados, observa-se que o percentual de pessoas do sexo feminino com vulnerabilidade econômica é maior, sendo de 47,1\% em instituições públicas e 46,9\% em instituições privadas. Ao analisar o crédito estudantil percebe-se que cerca de $30 \%$ dos homens e $37 \%$ das mulheres que estudam em instituições privadas não recebem qualquer tipo de bolsa de estudo ou financiamento, embora o curso não seja gratuito. Por outro lado, há uma porcentagem de estudantes que participam do FIES, Fundo de Financiamento Estudantil, $(11,07 \%$ do sexo masculino e $10,7 \%$ do sexo feminino). Além disso, podem ser beneficiários do ProUni (Programa Universidade para Todos) - sendo integral (22,5\% do sexo masculino e $18,2 \%$ do sexo feminino), parcial $(22,8 \%$ do sexo masculino e $21,5 \%$ do sexo feminino) ou ambos ( $4 \%$ do sexo masculino e $4,1 \%$ do sexo feminino). 


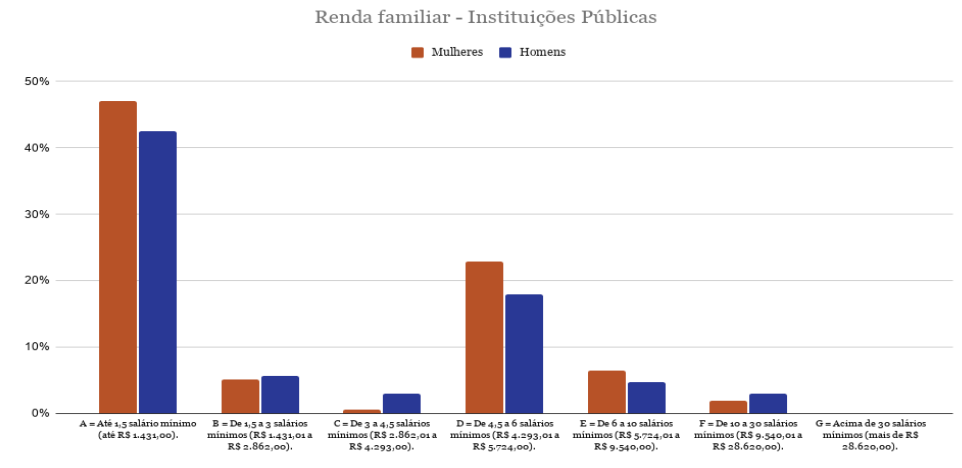

Figura 6. Percentual de mulheres e homens por renda mensal em instituições públicas

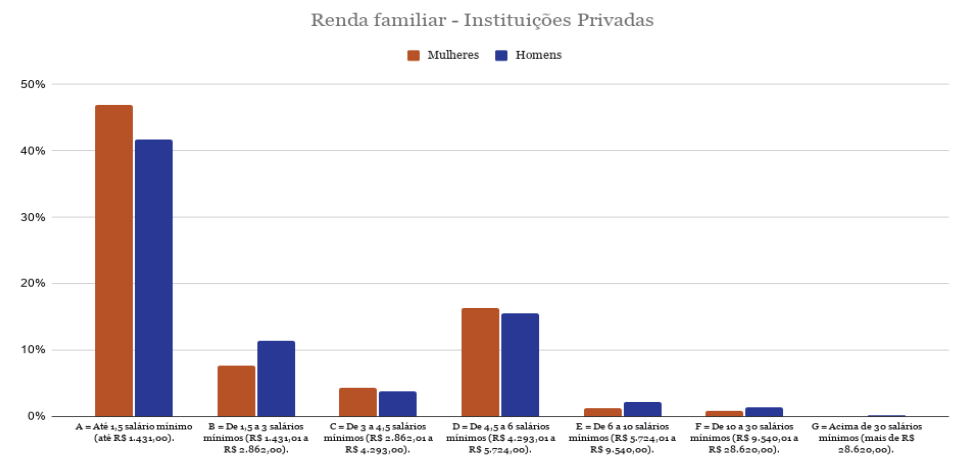

Figura 7. Percentual de mulheres e homens por renda mensal em instituições privadas

Para avaliar o tipo de escola que os estudantes da amostra cursaram o ensino médio, tem-se nas Figuras 8 e 9 que mostra o percentual de pessoas do sexo feminino e masculino agrupadas por cada tipo de escola em instituições públicas e privadas respectivamente. É possível verificar que, se comparado o percentual de estudantes de escola pública e privada em ambas as instituições, o percentual de pessoas do sexo feminino $(69,4 \%$ em instituição pública e $41,5 \%$ em instituição privada) advindas de escola pública é maior ao de pessoas do sexo masculino, outrossim, o percentual de pessoas do sexo masculino ( $11 \%$ em instituição pública e $5,9 \%$ em instituição privada) advindos de escola privada é maior ao de pessoas do sexo feminino.

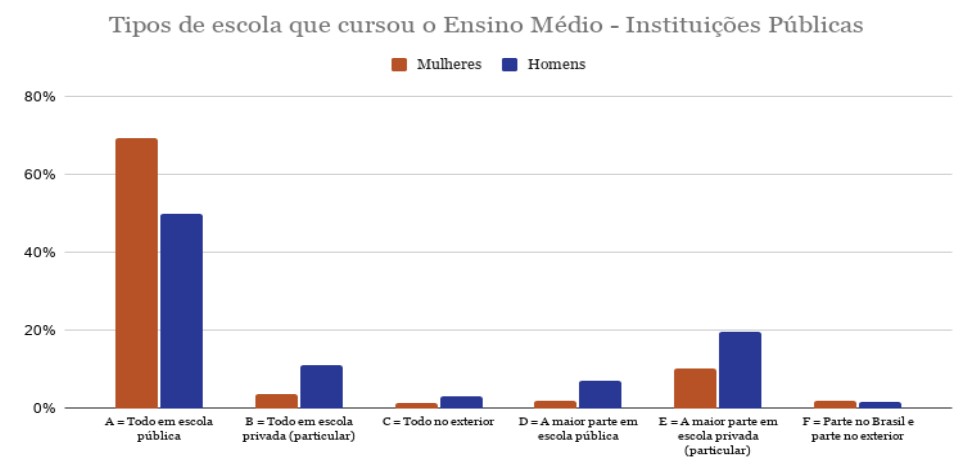

Figura 8. Percentual de mulheres e homens por tipo de escola que cursou o ensino médio em instituições públicas 


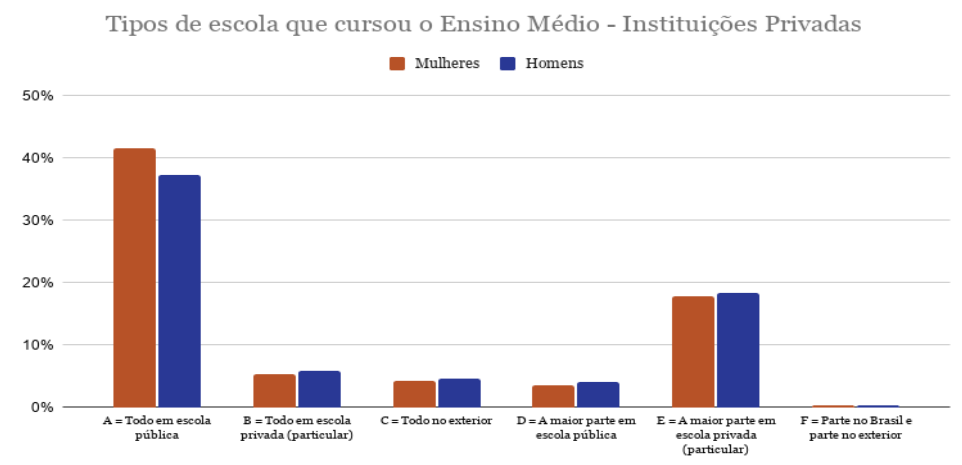

Figura 9. Percentual de mulheres e homens por tipo de escola que
cursou o ensino médio em instituições privadas

Para investigar o motivo de escolha de curso por parte dos estudantes da amostra, avalia-se tal ponto relacionado ao sexo dos participantes na Figura 10. Constata-se que a inserção no mercado de trabalho é o motivo de escolha do curso da maioria das pessoas do sexo feminino $(31,3 \%)$ e, além da inserção no mercado de trabalho, a vocação aparece como motivo de escolha de curso para a maioria das pessoas do sexo masculino ( $21,8 \%$ e $20,6 \%$ respectivamente).

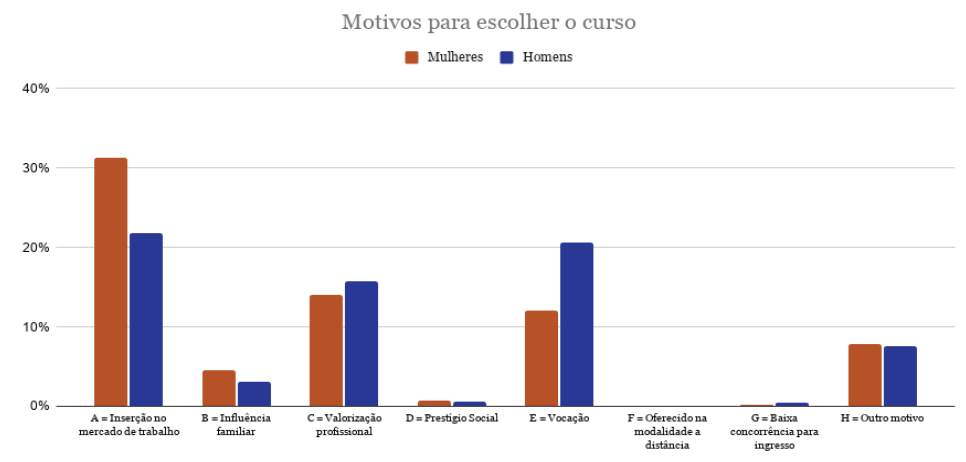

Figura 10. Percentual de mulheres e homens por motivos de escolha de curso

\section{Análise dos Dados}

Neste artigo foram analisados os microdados do Inep correspondente ao ENADE para relacionar fatores apresentados na seção 3 ao sexo e ao tipo de instituição. Quando se avalia uma amostra de estudantes que realizou o ENADE pode-se verificar o perfil de concluintes, ou seja, pessoas que persistiram em seus cursos e que serão futuros profissionais da área. Essa avaliação é de extrema importância para estudos que apontem propostas efetivas para a situação de desequilíbrio de gênero nas áreas STEAM, seja dentro das instituições de ensino e/ou no mercado de trabalho.

Visto que a evasão é uma situação recorrente nos cursos STEM como apresentado por Filho et al (2020) e Geglio (2018), os dados resultantes desse artigo poderiam ser relacionados aos dados de egressos desses cursos na região estudada.

Considerar o tipo de motivação para escolha de curso nos traz a reflexão apresentada por Casagrande et al (2016). O problema muitas vezes está na raiz, ou seja, no processo de socialização que ainda é diferenciada para meninos e meninas em sua infância e/ou adolescência. A maioria das mulheres acaba sendo ensinada a ajudar e ser ajudada, enquanto que a maioria dos homens é ensinada a ser autoconfiante, autônoma e 
competitiva. Assim, dados relativos à motivação da permanência desses estudantes, além da escolaridade/profissão dos pais contribuiria para que o estudo fosse aprofundado nesse âmbito.

O fato de a amostra analisada não apresentar nenhuma representante feminina indígena nas instituições públicas de um estado onde 4,84\% da população é indígena, cujo direito ao acesso à universidade é resguardado pela Lei de Cotas é algo a ser analisado em futuras pesquisas.

Filho et al (2020) mostram a visão da autora Olga Khazan sobre a necessidade da mudança nos currículos e na forma de ensinar nas áreas STEM para que esses cursos sejam mais atraentes para mulheres. Segundo ela, de acordo com dados estatísticos internacionais, os países com maior igualdade de gênero apresentavam menos mulheres nas profissões STEM, dessa forma, o problema pode não somente estar na socialização, mas também na forma como os cursos STEAM são apresentados - sendo frios e distantes da sociedade.

O fato, apresentado na seção 3 desse artigo, de que o percentual de homens que cursaram a maior parte de seu ensino médio em escola particular, tanto de instituição privada quanto pública, é maior se comparado com o percentual de mulheres na mesma condição, abre prerrogativas para avaliações mais significativas. Será que mulheres de escolas particulares estão tendo pouco incentivo em prosseguir em áreas STEAM em relação a outras áreas? Existem áreas que ainda são super valorizadas dentro de escolas particulares por razão da concorrência dos cursos em processos de seleção para universidades? Tais questionamentos podem ser avaliados em futuras pesquisas através de entrevistas com estudantes do ensino básico.

\section{Considerações Finais}

A análise de microdados do ENADE traz um perfil interessante de profissionais que estão entrando no mercado oriundos de cursos de STEAM. Mas além de dados meramente quantitativos como percentuais de concluintes por gênero, seu questionário socioeconômico nos dá um panorama mais amplo a ser avaliado.

Os resultados demonstraram que os homens compõem a maioria dos participantes do ENADE e então concluintes dos cursos STEAM no estado do Amazonas. A situação não muda quando analisamos os cursos de forma individual, com exceção do curso de Design em instituições públicas.

O motivo de escolha da maioria dos homens ficou dividido entre inserção no mercado de trabalho e vocação, ocorrência já apontada em outros trabalhos como o do autor Freitas et al (2019). Isso demonstra que o estigma de que mulheres não detêm a vocação para a área de exatas ainda é presente em nossa sociedade e precisa ser combatido através da socialização de meninos e meninas liberta de qualquer estratificação por gênero.

Outro fator que chamou a atenção, foi o fato da falta de representatividade da mulher indígena, chegando a zero na amostra referente a instituição pública. Bergamaschi et al (2018) traz uma possível explicação para essa inconsistência que nasce na limitação da Lei de Cotas $n^{\circ} 12.711$ de 29 de agosto de 2012, já que esta garante apenas o direito formal do indígena ao acesso ao ensino superior. É necessário que se desenvolvam ações de apoio à permanência desses povos, ainda mais nos casos em que a questão racial se une à questão de gênero.

Foi observado que o percentual de mulheres com vulnerabilidade econômica é maior que o percentual masculino, fato que quando associado ao dado de que a maioria das mulheres advém de escolas públicas pode também explicar o motivo de escolha de curso apresentado pela maioria delas: inserção no mercado de trabalho. Além disso, tal ocorrência abre prerrogativas para questionamentos quanto ao nível de incentivo escolar 
em relação a inserção de alunos e alunas nos cursos STEAM em escolas privadas.

\section{Agradecimentos}

Esta pesquisa, realizada no âmbito do Projeto Samsung-UFAM de Ensino e Pesquisa (SUPER), nos termos do artigo 48 do Decreto $\mathrm{n}^{\circ}$ 6.008/2006 (SUFRAMA), foi parcialmente financiada pela Samsung Eletrônica da Amazônia Ltda., nos termos da Lei Federal $\mathrm{n}^{\circ} 8.387 / 1991$, por meio dos convênios 001/2020 e 003/2019, firmados com a Universidade Federal do Amazonas e a FAEPI, Brasil.

\section{Referências}

Bergamaschi, Maria Aparecida; Doebber, Michele Barcelos; Brito, Patricia Oliveira. (2018). Estudantes indígenas em universidades brasileiras: um estudo das políticas de acesso e permanência. Rev. bras. Estud. pedagog. Brasília, v. 99, n. 251, p.37-53.

Carvalhaes, Flavio; Ribeiro, Carlos Antônio Costa. (2019) Estratificação horizontal da educação superior no Brasil: Desigualdades de classe, gênero e raça em um contexto de expansão educacional. Tempo Social, revista de sociologia da USP, v. 31, n. 1.

Casagrande, Lindamir Salete; Souza, Ângela Maria Freire de Lima e. (2016). Para além do gênero: mulheres e homens em engenharias e licenciaturas. Estudos Feministas, Florianópolis. http://dx.doi.org/10.1590/1806-9584-2016v24n3p825.

Colombo, Daniel Gama e. (2018). Análise do Perfil dos Ingressantes de Cursos de Mestrado e Doutorado. Cadernos de Estudos e Pesquisas em Políticas Educacionais. http://dx.doi.org/10.24109/9788578630669.ceppe.v1a8.

Filho, Roberto Leal Lobo e Silva; Lobo, Maria Beatriz de Carvalho Melo; Chagas, Renata Lucia Cavalca Perrenoud; Chagas, Joselito Moreira. (2020). Evasão e Valor Agregado nas IES de Engenharia no Brasil.

Freitas, Bárbara Santos; Cosme, Luciana Balieiro; Nascimento, Mayara Assis. (2019). Exame Nacional de Desempenho de Estudantes (ENADE) - Análise do Perfil das mulheres dos cursos da área de computação. Instituto Federal do Norte de Minas Gerais - IFNMG Montes Claros.

Geglio, Paulo César. (2018). Quem são os Estudantes da Carreira de Magistério?. Rev. FAEEBA - Ed. e Contemp., Salvador, v. 27, n. 53, p. 120-140.

Nasu, Vitor Hideo. (2020). Investigação Empírica Acerca da Relação entre Sexo e Desempenho Acadêmico na Educação Contábil. Universidade de São Paulo. São Paulo-SP, Brasil.

Tomás, M C. (2019). Percepções sobre a prova do ENADE sob a perspectiva de gênero e raça: uma análise dos cursos de Direito, Psicologia, Administração e Ciências Econômicas, 2015.

Zucatelli, C. (2019). Perfil socioeconômico, motivações e aspirações profissionais de concluintes da área de Educação do ensino superior no Brasil, 2014. XXI Encontro Nacional de Estudos Populacionais, Poços de Caldas-MG. 